Erneuerbare Energien, externe Effekte und ökonomische Bewertung

\title{
Nobody is perfect!
}

\section{Erneverbare Energien haben ein neves, zugleich uraltes Problem. Durch den Konflikt mit Naturschutzzielen zeigt sich, das sie ebenso externe Kosten verursachen wie fossile Brennstoffe. Unter dieser Prämisse sollte der Einsatz erneverbarer Energien neu überdacht werden.}

$\mathrm{D}$

Von Alexandra Dehnhardt und Ulrich Petschow

ie Nutzung fossiler Energieträger half die Knappheit der natürlichen Ressourcen industriell zu überwinden, führte aber auch zu Veränderungen in allen Bereichen der Natur, von denen momentan die Klimaproblematik am meisten Beachtung erfährt (1). Die Nutzung führt zur Inanspruchnahme der Senkenfunktion der Atmosphäre mit negativen Folgewirkungen an unterschiedlichen Schutzgütern. Die Nutzung der Senkenfunktion geschah bislang im Wesentlichen kostenfrei, die Emittenten tragen mithin nicht alle Kosten, die mit der Nutzung fossiler Energieträger verbunden sind.

Insofern ist die Argumentation der Befürworter Erneuerbarer Energien gerechtfertigt, dass die Nutzung fossiler Energien quasi subventioniert wird und von daher ungerechtfertigte Kostenvorteile eintreten, die es den erneuerbaren Energien schwer machen konkurrenzfähig zu werden. Entsprechend beruhen ökonomische Bewertungsansätze wie die sogenannten externen Kosten im Wesentlichen darauf, dass die anfallenden Kosten nicht in den Preismechanismus einbezogen sind und von daher Fehlallokationen die Folge sind.

\section{- Beschlussache Kosten}

Die Leitidee dieses Konzeptes ist im Grundsatz präferenzbasiert. Die Bestimmung externer Kosten gestaltet sich allerdings schwierig. Zum einen müssen die Schäden, die beispielsweise durch fossile Energieträger verursacht werden, identifiziert und quantifiziert werden, was vor allem eine Analyse der Ursache-Wirkungsbeziehungen voraussetzt, aber auch die Entwicklung von Vorstellungen, was zu erhalten ist. Zum anderen ist die Frage zu klären, wer als Verursacher zu bezeichnen ist und an welcher Stelle eine Kostenanlastung erfolgen sollte.

Typische Konzepte dabei sind die Schadenskosten und die Vermeidungskosten. Bei beiden Konzepten besteht erheblicher Diskussionsbedarf. Bei den Schadenskosten geht es vor allem darum zu identifizieren, welche Schäden einzubeziehen sind und welche Kosten dazu anzusetzen sind. Hier besteht im Grundsatz wenig Einigkeit, beispielsweise wie ein Menschenleben monetär zu bewerten ist. Zwar wird die Monetarisierung hinsichtlich der damit verbundenen ethischen Fragestellungen kritisiert, zugleich finden Entscheidungen darüber aufgrund anderer Kriterien statt, deren Moralität letztlich offen ist. Die Vermeidungskosten sind hingegen problemloser zu identifizieren. Hier wird von einem gegebenen Kapitalstock ausgegangen und vor dem Hintergrund der technischen und wirtschaftlichen Entwicklungstendenzen die Frage gestellt, welche Kosten in welchem Sektor durch Vermeidungsmaßnahmen entstehen. Unter den jeweiligen Annahmen lassen sich dann prioritäre Handlungsmaßnahmen identifizieren.

\section{- Motive für erneverbare Energien}

Aus herkömmlicher ökonomischer Sicht ergibt sich aus dem Klimawandel und dem Konzept der externen Kosten noch kein Hinweis darauf, dass erneuerbare Energien gefördert werden müssten. Die zentrale Strategie, die externen Kosten den Kohlendioxid-Emittenten anzulasten, hätte steuernde Wirkungen, die auch zu der Stärkung der Wettbewerbsfähigkeit der erneuerbaren Energien beitragen würden.

Mit der Art der Förderung der erneuerbaren Energien durch das Erneuerbare Energien Gesetz (EEG) wird allerdings nicht allein die Internalisierung der genannten externen Kosten angestrebt, sondern sie beinhaltet ganz wesentlich auch eine industriepolitisch motivierte Strategie, die darauf abzielt, die erneuerbaren Energien und die entsprechenden neuen Technologien wettbewerbsfähig zu machen.

Die Konzepte von Innovationssystemen und Pfadabhängigkeiten verweisen darauf, dass die Wirkung veränderter relativer Preise beispielsweise durch Internalisierung - vor allem in lock-in Situationen
- auf die Anreize zur Entwicklung alternativer Energien begrenzt ist. Die Erschließung der technischen Potenziale ist dem zu Folge allein durch den Preismechanismus nicht möglich.

Im Grundsatz wird mit der Förderung der erneuerbaren Energien eher versucht, first mover Vorteile zu erschließen und Zeitfenster zur Pfadgestaltung zu nutzen, um so die Diffusion der Technologien und Kostenreduktionen durch die Erschließung von economies of scale zu erreichen. Erneuerbare Energien setzen sich aus einem Portfolio unterschiedlichster Technologien zusammen, deren Wirkungen hinsichtlich der Emissionsvermeidung gleich sind, deren weitere Umweltwirkungen aber jeweils differenziert zu betrachten sind. Mit dem Klimaschutz einerseits und Eingriffen in den Naturhaushalt andererseits stehen unterschiedliche Umweltziele zueinander in Konkurrenz. Die Abwägung der unterschiedlichen Ziele ist ausgesprochen komplex, da sie im Grundsatz inkommensurabel sind. Beispielsweise umfasst der mit der EU-Wasserrahmenrichtlinie herzustellende ,gute ökologische Zustand“ auch die Gewässerstrukturgüte. Ein Ausbau der Wasserkraft kann diesem Ziel entgegenstehen.

Im Rahmen einer Studie zu kleinen Wasserkraftwerken hat das IÖW aufgezeigt, dass die gesamtwirtschaftlichen Kosten der Förderung kleiner Wasserkraftwerke deutlich höher sind als der gesamtwirtschaftliche Nutzen (2). In der Regel ist die betriebwirtschaftliche Rentabilität nicht gegeben, auch nicht bei Berücksichtigung der vermiedenen externen Kosten auf der Basis der Vermeidungskosten. Darüber hinaus führt der Ausbau der Wasserkraft selbst wieder zu Umweltwirkungen, deren monetäre Bewertung die Wirtschaftlichkeit weiter in Frage stellt. Da in der Studie mit Vermeidungskosten argumentiert wurde, kann zukünftig der Bau von kleinen Wasserkraftwerken aus Sicht des Klimaschutzes als effizient angesehen werden, dies aber erst dann, wenn eine Vielzahl von anderen Maßnahmen bereits erschlossen wurde.

\section{Zielkonflikte und Bewertung}

In einer aktuellen Studie wurden naturschutzoptimierte Szenarien zum Ausbau der erneuerbaren Energien entwickelt, die Restriktionen wie Schutzgebietsausweisungen berücksichtigen (Landschaftsschutz, Natura 2000 etc.) (3). Dies bedeutet, dass alle Gebiete, die bislang nicht unter einen Schutzgebietsstatus fallen, grundsätzlich für die Nutzung von erneuerbaren Energien zur Verfügung stehen sollten. Mit den Anreizstrukturen, die 
durch das EEG gesetzt werden, sind aber massive Veränderungen von Natur- und Landschaft und mithin auch Konflikte zu erwarten. Natur- und Landschaft haben bislang in den meisten Bewertungsstudien nur begrenzt eine Rolle gespielt, sowohl im Hinblick auf herkömmliche Technologien als auch auf erneuerbare Energien (4). Externe Kosten von erneuerbaren Energien sind auf zweierlei Weise zu problematisieren: zum einen führt der Klimawandel an sich zu erheblichen Auswirkungen auf Natur und Landschaft, das heißt die Vermeidung und Verringerung der Auswirkungen des Klimawandels kommt im Grundsatz auch Natur und Landschaft zu Gute. Zum anderen ist aber auch darauf zu verweisen, dass die erneuerbaren Energien Wirkungen auf Naturund Landschaft und gar auf Gesundheit haben können. Sie generieren damit selber externe Kosten, die durchaus einer ökonomischen Bewertung zugänglich sind. Hierbei handelt es sich um ein komplexes Abwägungsproblem.

Im Grundsatz wird mit dem Ausbau der erneuerbaren Energien die Knappheit von Natur und Landschaft noch einmal verstärkt und auch solche Bereiche unter Nutzungsdruck gesetzt, die, wie beispielsweise Gewässer, gerade eine gewisse Entlastung von landwirtschaftlichen Nutzungen erfahren haben. Die Bewertung dieser Knappheiten ist dann letztlich den erneuerbaren Energien als Kosten zuzuschreiben, die von ihnen nicht getragen werden. Diese Argumentation geht davon aus, dass es Präferenzen der Bevölkerung bezüglich Natur und Landschaft gibt, die noch nicht durch entsprechende rechtliche Regulierungen, wie die Ausweisung von Naturschutzgebieten abgedeckt sind. Die gesellschaftliche Akzeptanz, die beispielsweise das zur Verfügung stehende Potenzial der Windenergienutzung begrenzen kann, ist eng mit den Präferenzen der Bevölkerung verbunden und diese können auch als Kosten der EE begriffen werden.

Unter der Berücksichtigung präferenzbasierter ökonomischer Bewertungsverfahren aber auch regulierungsbezogener Verfahren könnte es das Ergebnis sein, dass die Einbeziehung der externen Kosten erneuerbarer Energien andere Optionen der Kohlendioxid-Reduktion ,wirtschaftlicher" macht, als es bei den gegenwärtigen Berechnungen der Fall ist. Vor diesem Hintergrund muss die Argumentation, die Klimaschutzziele könnten ohne den Ausbau von erneuerbaren Energien nicht erreicht werden, neu betrachtet werden. Dies ist kein Gegenargument zum Klimaschutz, es verweist allein darauf, dass die pla- nerische Berechnung von Kohlendioxid-Minderungspotenzialen durch den Ausbau der erneuerbaren Energien durchaus auf den Prüfstand gestellt werden sollte. Der zum Teil vorhandene Widerstand gegen erneuerbare Energien ist keineswegs allein oder gar hauptsächlich von den Energiekonzernen mobilisiert, sondern wird durch eine Vielzahl von UmweltschützerInnen und normalen BürgerInnen getragen. Zudem ist nicht zu verkennen, dass mittlerweile die ökonomischen Interessen zur Durchsetzung der erneuerbare Energien zunehmen.

\section{Fazit}

Die Auswirkungen der erneuerbare Energien sind kaum auf genereller Ebene, sondern nur kontextabhängig zu bewerten: Dem ,globalen“ Gut Klimaschutz stehen hochgradig heterogene Umweltgüter auf der regionalen und nationalen Ebene gegenüber, die nur begrenzt aggregierbar sind. Die Balance herzustellen zwischen den unterschiedlichen Effekten der erneuerbare Energien ist zentral, die planerisch vielfach vermeidungskostenorientierten Ansätze erweisen sich aus ökonomischer Sicht als nicht hinreichend konsequent. Die naturschutz- und landschaftsorientierten Präferenzen sollten herangezogen werden, um den Umfang des Ausbaus zu überprüfen und zugleich einen Vergleich zu den Vermeidungskosten in anderen Bereichen herstellen zu können.

\section{Anmerkungen}

(1) Sieferle, R. P. : Der unterirdische Wald. Energiekrise und Industrielle Revolution. München 1982.

(2) Meyerhoff, J./ Petschow, U.: Umweltverträglichkeit kleiner Wasserkraftwerke. Zielkonflikte zwischen Klima- und Gewässerschutz. UBA Texte 13/98, Berlin 1998.

(3) Siehe Anmerkung 1, Seite 14: Ökologisch optimierter Ausbau der Nutzung erneuerbarer Energien in Deutschland. Berlin 2004, siehe auch Beitrag von Nitsch et al. in diesem Heft.

(4) European Commission (Ed.): ExternE, Externalities of Energy. Brussels 1995

\section{Die Autorlnnen}

Alexandra Dehnhardt ist wissenschaftliche Mitarbeiterin im, Ulrich Petschow Leiter des Forschungsfeldes Umweltökonomie und -politik am Institut für ökologische Wirtschaftsforschung (IÖW)

Kontakt: IÖW, Potsdamerstr. 105, 10785 Berlin. Tel. 030-8845840, E-Mail: ulrich.petschow@ioew. de, alexandra.dehnhardt@ioew.de

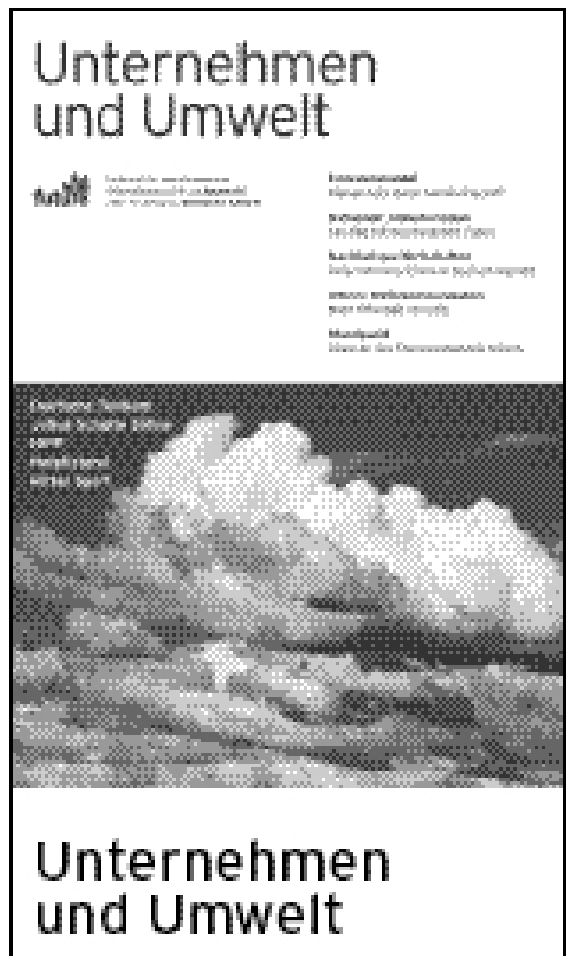

... ist die Zeitschrift für umweltorientieste Unternehmers politik von future e.V.

... bietet Fachinformationen zum Urrweltschutz und Best-Practice- Beispiek aus Unternehmen.

... bietet viermal im lahr Urternehmersbeipiele, Literatur- und Verantaltungshinweise.

Mit enem Schwerpunktthema in jedem Heft. Zum Beispiel:

2/04: Envissionshundel

1/04: Bildung fiv Nachimaltige Entwrickhng

4/03: Kompetern nocthaltig entwicke in

3/03: Nacthaltigke it und Region

2103: Kapital fir Nacthaltighert

1/03: Energieetficienz

3-4/02: Benchururking for Sustainability

2102: Ohomurhetring

1/102: Nachisaitigkeit und Gewerbebus

4101: Nacthaitigkeit im Handwerk

3/01: Klimaschutz

Bestellen sie ihr kostenloses

Probe-Abo im Internet unter

$w w w$. fut ure-ev.de

future e.V. Būro Bochum

Afrl Varerholt 123

44797 Bochum

Fon: 0234/9799513

Fax: 0234/9799514

bochum fituture -ev.de

nww. future-ev.de

futuie 
(c) 20I0 Authors; licensee IÖW and oekom verlag. This is an article distributed under the terms of the Creative Commons Attribution Non-Commercial No Derivates License (http://creativecommons.org/licenses/by-nc-nd/3.o/), which permits unrestricted use, distribution, and reproduction in any medium, provided the original work is properly cited. 